\title{
A UTILIZAÇÃO DA MODELAGEM AMBIENTAL PARA SISTEMATIZAÇÃO DO CONHECIMENTO TÁCITO: IDENTIFICAÇÃO DE CORREDORES PREFERENCIAIS PARA LINHAS DE TRANSMISSÃO DE ENERGIA ELÉTRICA
}

The use of environmental modeling for systematization of tacit knowledge: identification of optimal corridor for transmission lines of electricity

\author{
PEDRO BUENO ROCHA CAMPOS 1 \\ MARCOS ANTÔNIO TIMBÓ ELMIRO² \\ RODRIGO AFFONSO DE ALBUQUERQUE NOBREGA ${ }^{3}$
}
${ }^{1,2,3}$ Universidade Federal de Minas Gerais - UFMG
${ }^{1,2,3}$ Instituto de Geociências - IGC

pbrcampos@gmail.com; timboelmiro@gmail.com; raanobrega@ufmg.br

\section{RESUMO}

A necessidade de expansão do setor elétrico requer o aprimoramento constante das formas de projetar e executar empreendimentos como linhas de transmissão de energia elétrica. Embora, a disseminação de tecnologias de geoinformação estejam auxiliando a modernização desse processo, um dos grandes desafios é preservar o conhecimento acumulado ao longo dos anos de desenvolvimento de projetos. Os padrões de execução de projetos passados podem ser encontrados nas paisagens em que os mesmos foram executados. A hipótese avaliada neste trabalho é a possibilidade de empregar geotecnologia para extrair o conhecimento tácito através de padrões de execução de projetos lineares desenvolvidos no passado por meio da análise da paisagem, e utilizar tais informações em um processo de analise multicriterial para auxiliar a modelagem novos corredores de transmissão de energia. Este procedimento representa a oportunidade de estabelecer termos comparativos e parametrizados para interfacear às novas tecnologias e ganho de produtividade para modernizar o planejamento do setor de distribuição de energia. A investigação se apóia na análise multitemporal. Para tanto, a elaboração de um modelo espacialmente explícito e dinâmico faz-se necessária visando reconhecer não só o padrão espacial de ocorrência do fenômeno, mas também a sua variação na 
história. O presente trabalho emprega metodologias robustas, como Peso de Evidências e Least Costly Path para estudar o padrão espacial da localização de linhas de transmissão de energia na paisagem. Os resultados demonstram a viabilidade do uso de geoprocessamento e análise da paisagem, bem como a possibilidade de identificação de variáveis de caráter geográfico não consideradas nas tratativas normativas do setor, além de indicarem os locais de preferência para locação destes empreendimentos,

Palavras-chave: Linhas de Transmissão de Energia Elétrica; Conhecimento Tácito; Análise da Paisagem, Análise Ambiental.

ABSTRACT
The need for expansion of the electricity sector requires constant improvement for designing methods and project developments, such as electricity transmission lines. The dissemination of geoinformation technology supports the modernization of the process, yet the preservation of tacit knowledge is a key challenge to be considered. The patterns resulting from projects implemented in the past can be found in the landscape. This paper hypothesizes the use of geotechnologies to extract the tacit knowledge from linear engineering projects printed in the landscape through landscape analysis, therefore use the information in a multicriterial decision framework towards modeling new corridors for transmission lines of electricity. This procedure can represent an opportunity for establishing metrics for interfacing new technologies and productivity gains to modernize the planning process of the sector of energy distribution. This research is based on a multi-temporal analysis. The model developed is dynamic and geographically explicit in order to recognize not only the spatial occurrence pattern of the phenomenon, but also the variation along their history. This study employs robust methodologies, such as Weight of Evidence and Least Costly Path to study the spatial pattern of the energy transmission lines implementation across the landscape. The results demonstrate the feasibility of GIS and landscape analysis, as well as the possibility of identifying geographic variables not considered in the existing regulation, and indicate preferable locations for new corridors.

Keywords: Electricity Transmission Lines; Tacit Knowledge; Landscape Analysis; Environment Analysis.

\section{INTRODUÇÃO}

A expansão do sistema de transporte de energia elétrica requer a construção de novas linhas de transmissão e distribuição de energia, as quais são empreendimentos de infraestrutura básica, necessários ao desenvolvimento regional, e que atendem à cadeia produtiva e econômica. Quanto mais cresce a demanda e a oferta de energia elétrica, mais questões surgem sobre a forma de implantação das redes de transmissão e distribuição de energia, bem como a determinação dos locais de construção destes empreendimentos (EPRI, 2006). Neste setor, há investimentos 
voltados ao desenvolvimento de tecnologias e sistemas para elaboração e execução destes projetos.

Dentre as pesquisas desenvolvidas, destacam-se as relacionadas à escolha e locação dos traçados de linha de transmissão de energia, como a metodologia desenvolvida pelo Electric Power Research Institute (EPRI, 2006), o trabalho apresentado por Schmidt (2009), bem como o estudo elaborado para a otimização de rotas para linhas de transporte de energia, que resultou no desenvolvimento do software Rota (BELÉM et al, 2009). Essas pesquisas têm como base a metodologia de análise multicritério para decisão do traçado e a associação com algoritmos de otimização das rotas fornecidas por essas análises.

Concomitante com o desenvolvimento de novas tecnologias, o projeto de uma grande obra de infraestrutura segue regras pré-definidas que o acompanham desde sua fase de planejamento até as fases de execução e operação, algo também inerente aos projetos de linha transmissão. São várias as normativas relacionadas a este tipo de empreendimento, entre elas tem-se a NBR 5.422 (ABNT, 1985), para projetos de linhas aéreas de transmissão de energia elétrica, e os Procedimentos de Distribuição de Energia Elétrica no Sistema Elétrico Nacional - PRODIST (ANEEL, 2013). Há também, as proposições teóricas para elaboração dos projetos, como a oferecida por Labegaliniet al. (1992).

Entretanto, a real implementação do projeto não está totalmente descrita nesse conjunto de regras e procedimentos. Grande parte do conhecimento necessário para a implementação pode ser classificado como conhecimento tácito, e este é inerente à experiência dos profissionais envolvidos no projeto (RIBEIRO, 2012). Segundo Polanyi (1966), o conhecimento tácito pode ter, entre suas diversas distinções e aprofundamentos, a definição simplista de saber mais do que se consegue falar ou transmitir. Conforme Ribeiro (2012), a sistematização do conhecimento tácito e da experiência dos profissionais tem oferecido grandes vantagens e aumento de produtividade para as empresas. Em geral, o conhecimento tácito acumula a experiência desenvolvida de profissionais atuando ao longo do tempo em diversas áreas dentro de um mesmo projeto. Portanto, a sistematização desse tipo de conhecimento tem a complicada tarefa de apreender a forma como foi realizado algo que nem mesmo quem o executou tem a habilidade de explicar em termos parametrizáveis, ou que não sejam subjetivos ao ponto de variar bruscamente entre diferentes profissionais.

A possibilidade de sistematização desse conhecimento torna-se, então, imprescindível ao intuito de aprimorar as técnicas e metodologias de locação das linhas de transmissão de energia. O presente trabalho propõe, para tanto, uma metodologia de identificação de padrões espaciais na implantação de projetos de obras de infraestrutura de transmissão e transporte de energia elétrica. Para o estudo, foram selecionadas linhas de transmissão de energia construídas entre 1960 e 2000, e a investigação teve como objetivo a busca por padrões espaciais expressos na paisagem destas feições lineares, bem como a aplicação desses padrões na previsão 
de projetos futuros. As variáveis envolvidas foram modeladas e analisadas em um sistema multicriterial de suporte à decisão.

\section{FUNDAMENTAÇÃO DO MODELO}

Quando são utilizadas metodologias de análise multicritério na fase de planejamento do projeto, parte do conhecimento tácito pode ser capitalizado e aproveitado. As metodologias de análise multicritério possibilitam a combinação de diferentes variáveis, atribuindo-se a cada uma delas pesos específicos de ponderação. A boa prática sugere que as regras de uso de cada variável devam ser estabelecidas por um conjunto de profissionais, em geral especialistas nas variáveis e nos respectivos cenários em análise, os quais são em geral confrontados numericamente no sistema multicriterial de suporte à decisão (SADASIVUNI et al., 2009).

A ponderação das variáveis tem como base a inserção de uma escala de preferência ou impacto, positivo ou negativo, para cada variável. O modelo descrito por Saaty (1980) propõe uma organização hierárquica para as comparações entre as variáveis através de cenários. O modelo é denominado Analytical Hierarchy Process (AHP) e, embora tenha sido desenvolvido para auxiliar decisões econômicas, também tem sido aplicado para apoiar projetos de engenharia, por ser um processo robusto, confiável e de fácil implementação (MUNIER, 2011, NOBREGA 2009). Nobrega (2009) e Sadasivuni (2009) demonstram a modelagem do método AHP em ambientes SIG (Sistema de Informações Geográficas) como solução para o suporte ao planejamento de corredores de transporte e obras de infraestruturas lineares que demandem análise espacial.

No modelo AHP os pesos das variáveis não são atribuídos diretamente, mas sim através de parâmetros de preferência. A parametrização do processo conta com uma escala com fatores variando entre 1 (igual preferência) e 9 (menor preferência), para serem utilizados em uma matriz de comparação pareada a cada duas variáveis. O método permite calcular o ranking de forma indireta através de autovalores da matriz e uma análise final de consistência para assegurar a compatibilidade dos valores de entrada. $\mathrm{O}$ emprego de metodologias de análise multicritério para solução de decisões críticas, como a AHP, tem como principal objetivo proporcionar a solução de problemas entre as variáveis, cenários e opiniões conflitantes no processo de tomada de decisão em um projeto, como por exemplo, viabilizar a otimização de recursos disponíveis, e ao mesmo tempo minimizar os impactos ambientais (NOBREGA, 2009).

Ao ser introduzida uma classificação de preferências podem surgir problemas de interpretação sobre o que é maior ou menor para cada profissional (EPRI, 2006). Contudo, técnicas são aplicadas para minimizar o desvio entre as classificações e gerar um consenso para a escala aplicável a cada variável. Entretanto, mesmo em consenso, esta escala parte da subjetividade da capacidade de interpretação pessoal.

A interpretação pessoal esta também impressa na paisagem por projetos executados por profissionais. Segundo Ribeiro (2012), a capacidade de interpretação 
desse conhecimento tem relação direta com a experiência adquirida no acúmulo dos projetos executados. Portanto, a possibilidade de extrair a escala de classificação das variáveis do que de fato foi executado pelo profissional ou pela equipe responsável por um projeto pode reduzir a subjetividade da informação, gerando termos comparativos e parametrizados. Em outras palavras, a análise do padrão de execução dos projetos de uma mesma empresa ao longo do desenvolvimento de sua experiência pode auxiliar na busca pelo aperfeiçoamento dos planejamentos e das metodologias de locação dos empreendimentos.

Para tanto, é possível através da modelagem dinâmica, investigar o comportamento de padrões espaciais expressos na cobertura e uso do solo ao longo do tempo e, com isso, criar uma base de conhecimento que pode ser utilizada para apoiar modelos de previsão. A modelagem dinâmica tem a capacidade de representar a realidade de forma sintética em modelos análogos e abstratos (CHORLEY e HAGGET, 1974). De acordo com Christofoletti (1998), a representação em um ambiente controlado do sistema de forças e energias que interagem entre si fornece parâmetros para variáveis que explicam ou que determinam o funcionamento de determinado fenômeno, como por exemplo, a existência de um acontecimento num lugar específico, ou seja, que obedeça a um padrão espacial.

O estudo sobre a identificação de padrões espaciais tem larga aplicação em pesquisas sobre mudança de uso e cobertura do solo, conforme abordagem nos trabalhos de Macedo et al (2013), Soares-Filho (1998), Mendes e Canales (2011) e Almeida et al (2003). Há também, muitas aplicações na identificação de padrões para localização de fenômenos, conforme Bonham-Carter (1994) e Nunes (2010). Portanto, se a existência de linhas de energia for considerada um fenômeno que ocorre na paisagem e determinado por variáveis mensuráveis é possível que os traçados das linhas de transmissão de energia obedeçam a padrões espaciais.

Para identificação dos padrões espaciais foi utilizada a metodologia proposta por Almeida et al (2003) e Bonham-Carter (1994), pois ambos os trabalhos têm como base o Teorema de Bayes, também denominado Teorema da Probabilidade Condicional, cuja aplicação fornece a probabilidade de ocorrer um fenômeno, nesse caso a existência de linhas de energia $(Z)$, dado a ocorrência de um conjunto de variáveis explicativas e relacionadas ao fenômeno $(\mathrm{B} \cap \mathrm{C} \ldots \cap \mathrm{N})$. Esta metodologia tem larga aplicação na identificação de padrões espaciais de variados fenômenos (BONHAM-CARTER, 1994; SOARES-FILHO, 1998; ALMEIDA et al, 2003; NUNES, 2010; MACEDO et al, 2013). Almeida et al (2003) descreve como Pesos de Evidência a influência das variáveis explicativas no fenômeno em análise e a base da metodologia está fundamentada nas equações de 1 a 6 :

$$
P(Z \mid B \cap C \ldots \cap N)=P(Z) \frac{P(B \cap C \ldots \cap N \mid Z)}{P(B \cap C \ldots \cap N)}
$$


A equação 1 encontra a probabilidade de ocorrência do fenômeno Z, mas para saber se há um padrão espacial é necessário verificar o comportamento das variáveis onde não ocorre Z, ou seja, uma probabilidade Z' conforme a equação 2:

$$
P\left(Z^{\prime} \mid B \cap C \ldots \cap N\right)=P\left(Z^{\prime}\right) \frac{P\left(B \cap C \ldots \cap N \mid Z^{\prime}\right)}{P(B \cap C \ldots \cap N)}=1-P(Z \mid B \cap C \ldots \cap N)
$$

A definição da presença ou ausência de linha de energia estabelece uma variável dependente binária, com comportamento de uma função logística quando variada por um conjunto de variáveis explicativas. Como uma função logística tem características não lineares de regressão, há a necessidade da linearização dessa função para que seja possível determinar a capacidade ou qual a participação de cada variável explicativa na presença ou ausência da variável dependente (MENDES E CANALES 2011). A linearização da função ocorre de uma forma simplista, quando o intervalo de valores possíveis da variável dependente deixa de ser binário, ou seja, assumir o valor 0 ou 1. A divisão da probabilidade de $\mathrm{P}(\mathrm{Z} \mid \mathrm{B} \cap \mathrm{C} \ldots \cap \mathrm{N})$ por $\mathrm{P}\left(\mathrm{Z}^{\prime} \mid \mathrm{B} \cap \mathrm{C} \ldots \cap \mathrm{N}\right)$ fornece a chance de ocorrência da variável dependente. Este conceito é denominado Odds (O) e tende ao $\infty$ quando a probabilidade de $\mathrm{Z}$ tende a 1 (Equação 3).

$$
O=\frac{P(Z \mid B \cap C \ldots \cap N)}{1-P(Z \mid B \cap C \ldots \cap N)}
$$

Dessa forma, o intervalo de variação passa a ser $0 \leq \mathrm{P}(\mathrm{Z} \mid \mathrm{B} \cap \mathrm{C} \ldots \cap \mathrm{N}) \leq+\infty$ e para eliminar o limite inferior utiliza-se uma transformação logit (logisticprobabilityunit) da Odds, conforme equação 4:

$$
\operatorname{Logit} O=\ln _{e}\left[\frac{P(Z \mid B \cap C \ldots \cap N)}{1-P(Z \mid B \cap C \ldots \cap N)}\right]=W_{N}^{+}
$$

Onde $W_{N}^{+}$representa o coeficiente do peso de evidência. Este termo assume a característica de atribuir um peso de explicação para cada variável independente, onde quanto maior o valor de $W_{N}^{+}$mais associada é a ocorrência de $\mathrm{Z}$ com a variável em análise. Quando todas as variáveis são analisadas em conjunto, atribuindo-se a cada uma seu respectivo coeficiente, encontra-se o peso de evidência, ou seja, a probabilidade de ocorrência de $\mathrm{Z}$ dado a ocorrência do conjunto $(\mathrm{B} \cap \mathrm{C} \ldots \cap \mathrm{N})$ de variáveis mensuradas na paisagem. Para tanto, o logit de $\mathrm{O}$ é convertido novamente para probabilidade e substituído na equação 1 , conforme as equações 5 e 6 :

$$
\frac{P(Z \mid B \cap C \ldots \cap N)}{1-P(Z \mid B \cap C \ldots \cap N)}=e^{\Sigma W_{N}^{+}}
$$

Substituindo os termos da equação 5 na equação 1 , tem-se a equação 6 que relaciona os pesos de evidência com a probabilidade de $P(Z \mid B \cap C \ldots \cap N)$ : 


$$
P(Z \mid B \cap C \ldots \cap N)=\frac{e^{\Sigma W_{N}^{+}}}{1+e^{\Sigma W_{N}^{+}}}
$$

A representação da paisagem considerou as variáveis que têm maior capacidade explicativa para a ocorrência de linhas de energia. Para tanto, foi considerada a norma técnica de especificação de obras desse tipo de empreendimento, NBR 5.422 (ABNT, 1985), a proposta teórica para projetos de linhas de transmissão de energia oferecida por Labegaliniet al (1992), bem como as pesquisas desenvolvidas no estudo de corredores ótimos para locação das linhas (EPRI, 2006; SCHMIDT, 2009). Não foram consideradas as variáveis que não são mensuráveis diretamente na paisagem pelo profissional quando do planejamento do empreendimento, como condição de fundação, direção e velocidade do vento, bem como áreas conflitantes com o transporte de energia e que não estão identificadas na paisagem.

Os estudos desenvolvidos pelo EPRI (2006) trazem inúmeras variáveis, classificadas em grupos a partir do conjunto de profissionais envolvidos com o projeto e conforme a etapa de desenvolvimento da metodologia proposta. Nesta pesquisa buscou-se identificar padrões espaciais de macro corredores preferenciais para linhas de energia, conforme a proposta metodológica do trabalho do EPRI (2006).

\section{MATERIAIS E MÉTODOS}

O modelo foi aplicado na região do Triângulo Mineiro no estado de Minas Gerais e parte do estado de Goiás. Os municípios abrangidos pela pesquisa foram Uberlândia, Araguari, Uberaba, Ituiutaba e Itumbiara, cobrindo uma área de 21.650 $\mathrm{km}^{2}$, ilustrada na Figura 1. As linhas de transmissão de energia pesquisadas são de responsabilidade da Cemig Distribuição S/A e Cemig Transmissão S/A, as tensões nominais variam entre $69 \mathrm{kV}$ e $500 \mathrm{kV}$. A maioria destas linhas de energia tem estrutura metálica de suporte ao cabeamento e faixas de segurança a partir do eixo central variável entre 23 e 40 metros.

De forma geral, a solução encontrada para resolução do problema levantado foi a elaboração de um modelo espacialmente explícito e multitemporal, visando encontrar a probabilidade de ocorrência de linha de transmissão de energia dado um conjunto de variáveis explicativas. O modelo é considerado multitemporal porque utiliza várias linhas de transmissão, divididas de acordo com o período de construção, para extrair o padrão espacial. Esta probabilidade, após a validação do modelo, será utilizada para traçar rotas para linhas de transmissão de energia, cuja otimização ocorrerá com a inserção de variáveis que restrinjam o traçado. O modelo foi elaborado nas plataformas Dinâmica Ego e ArcGis 10.1. 
Figura 1 - Mapa de localização da área de estudo.

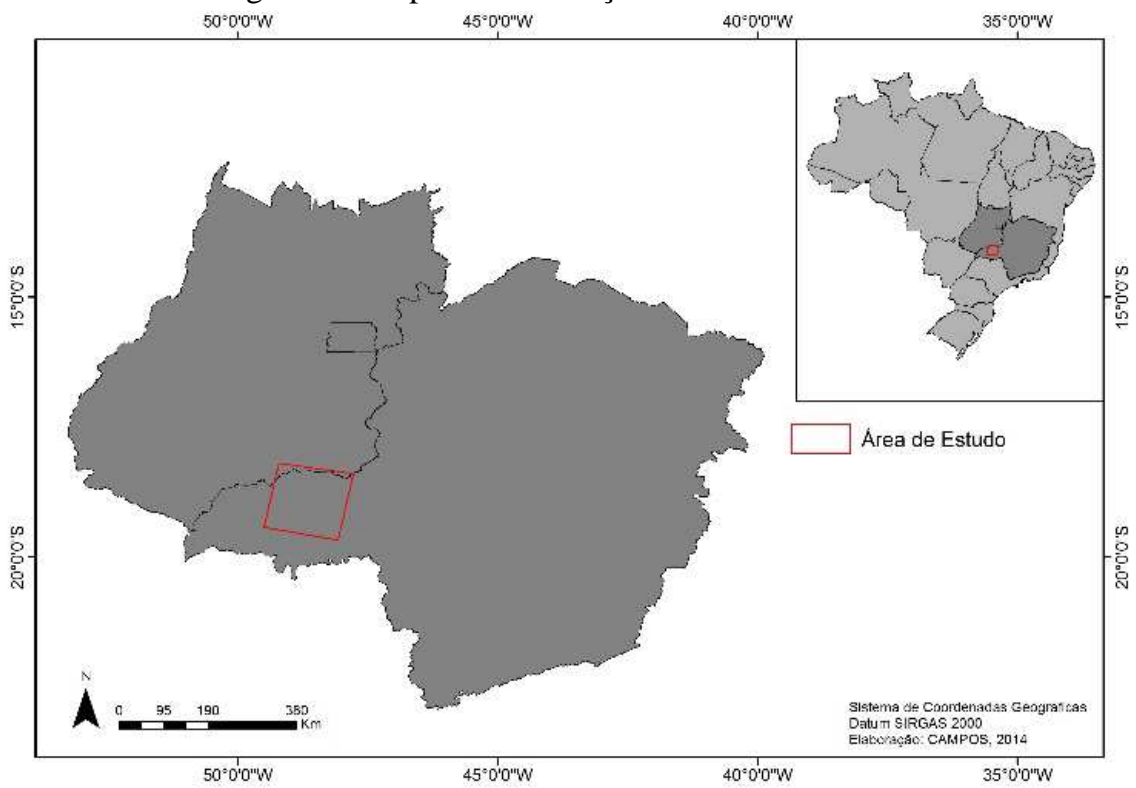

A metodologia foi dividida em quatro fases principais, sendo cada uma dependente da etapa anterior. A primeira fase consiste em realizar para cada período de tempo a categorização em classes das variáveis contínuas, a definição dos pesos de evidência e os mapas preliminares de probabilidade de ocorrência de linhas de transmissão de energia. A segunda fase tem por objetivo a calibração dos pesos de evidência e o recálculo dos mapas de probabilidade. Na validação do modelo, terceira fase, gera-se o traçado de uma linha já existente a partir do mapa de probabilidade de ocorrência de linha de transmissão. A quarta e última fase insere variáveis consideradas não observáveis na paisagem e que tem o caráter de otimizar o traçado. O fluxo representado na Figura 2 delimita e exemplifica a estrutura do modelo:

Optou-se pela utilização das variáveis: distância a rodovias, distância a linhas existentes, distância a principais rios e cursos de água, uso e cobertura do solo e declividade. Compreendem-se estas como o conjunto de variáveis explicativas da preferência pela ocorrência de linhas de transporte de energia em macro corredores preferenciais (EPRI, 2006; SCHMIDT, 2009). Já para a proposição de alternativas quanto ao traçado baseado no padrão espacial das linhas existentes foram consideradas variáveis não identificáveis na observação da paisagem no momento do planejamento do projeto, como áreas de restrição ambiental, concessão de lavras minerárias e assentamentos rurais, visto que os impactos socioambientais da 
interferência das linhas de energia nestas ocupações da superfície podem ser significativos (ABNT, 1985; LABEGALINI et al, 1992; EPRI, 2006).

Figura 2 - Esquema de Funcionamento do Modelo.

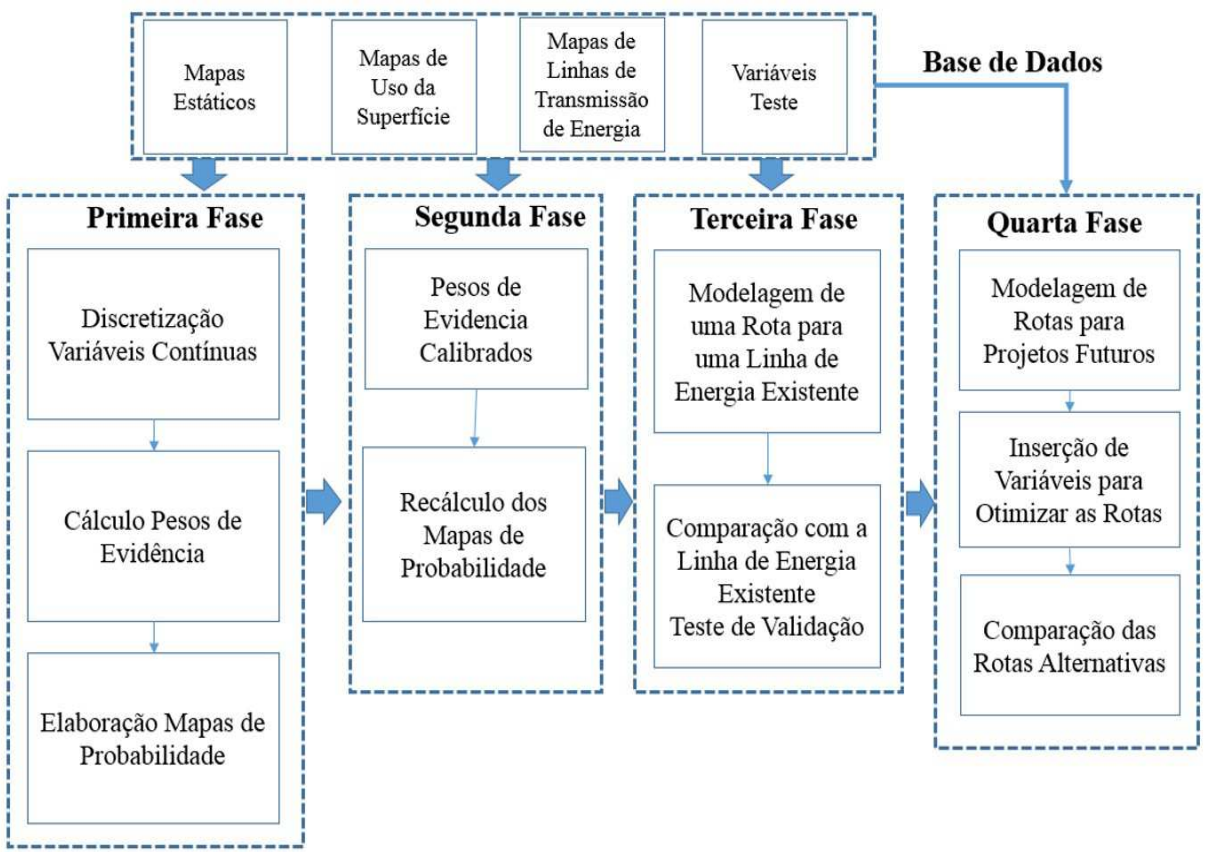

Todas as variáveis foram convertidas para a forma matricial de representação, onde cada célula da matriz tem 60x60 metros, com o mesmo par de coordenadas, sistema de coordenadas e DATUM. O mapa de uso e ocupação do solo foi extraído da classificação de imagens dos satélites Landsat 1, 5 e 7, adquiridas nos anos de 1975, 1984, 1990 e 2000. Os mapas foram classificados em cinco classes, sendo estas, urbano, silvicultura, vegetação nativa, corpos de água e agropecuária.

As linhas existentes foram divididas em cinco categorias, conforme o ano de entrada em operação. A primeira classe refere-se às linhas construídas antes de 1975, e as demais classes são referentes aos intervalos dos mapas de uso e ocupação do solo, visto a disponibilidade das imagens dos satélites Landsat. As demais variáveis foram consideradas constantes no modelo, ou seja, não possuem variação no tempo.

O modelo é iterativo e tem um ciclo de repetição para cada categoria de linhas existentes, sendo consideradas, em cada iteração, as linhas que já existiam no período anterior ao que está em análise, mais as linhas que foram construídas no período em questão. Dessa forma, é possível a análise da variação do fenômeno de 
ocorrência de linhas na paisagem. O modelo tem a capacidade de verificar quais as características dos lugares onde já existiam linhas e quais são as características dos locais onde foram construídas novas linhas, analisando se há algum padrão espacial nos locais onde foram construídas novas linhas de energia. Para cada iteração o modelo calcula a distância euclidiana para as linhas já existentes e seleciona qual o uso e ocupação da superfície referente ao período considerado na iteração.

Para o cálculo da probabilidade foi utilizada a metodologia de Pesos de Evidência (ALMEIDA et al, 2003; BONHAM-CARTER, 1994). Para a categorização das variáveis com valores contínuos foi utilizada a separação dos intervalos das classes através da metodologia proposta por Agterberg e Bonham Carter (1990) e adaptada por Soares-Filho et al (2008) no programa Dinâmica Ego. Este é um processo para discretizar as variáveis contínuas em classes, considerando os intervalos em que a ocorrência e a não ocorrência do fenômeno são mais significativas. A aplicação desta metodologia pode ser observada nos trabalhos de Almeida et al (2003) e Nunes (2010).

A calibração do modelo consiste na verificação da independência entre as variáveis. Para tanto, foram realizados dois testes estatísticos: o cálculo do Coeficiente de Cramer e do índice Joint Information Uncertainty (BONHAM CARTER, 1994). Os valores destes testes estatísticos são adimensionais e são utilizados para verificar se existe dependência entre as variáveis utilizadas, ou seja, se duas ou mais variáveis estão explicando a mesma coisa. As variáveis são comparadas par a par e os valores são apresentados para cada comparação. Quanto menores os valores encontrados, menor é a dependência entre as variáveis. Bonham Carter (1994) sugere o limite de 0,5 para as duas análises. É ainda parte da calibração do modelo o ajuste dos pesos de evidência, garantindo que a função de variação dos coeficientes em relação à alteração de cada variável apresentasse uma correlação. Os coeficientes que não descreviam nenhuma função ou apresentavam baixa correlação com as variáveis foram desconsiderados.

A validação foi feita a partir da locação de uma rota para uma linha existente que não foi considerada no modelo. A locação utilizou a metodologia aplicada no trabalho de Soares-Filho et al (2008), que prevê a elaboração de um mapa representando uma superfície de custo para sair de qualquer ponto do mapa para um outro ponto específico, cujo custo é o valor da probabilidade de existência de linhas de energia. Dessa forma, é possível identificar o caminho entre dois ou mais pontos no mapa com maior probabilidade de existência de linhas de energia, ou seja, este procedimento possibilita a identificação dos corredores preferenciais à este tipo de empreendimento. Ao ser informado um ponto de origem o modelo calcula o custo acumulado de deslocamento de uma célula da matriz para outra, utilizando os valores de probabilidade. O algoritmo que faz esse cálculo é do tipo pushbroom que faz uma varredura de toda a superfície de custo e identifica os valores célula a célula da matriz a partir do ponto informado como origem, acumulando os valores conforme ocorre um distanciamento do ponto de origem. Com o mapa de custo acumulado calculado, é utilizado um algoritmo denominado Least Costly Path que 
encontra o menor custo para atravessar o mapa a partir de um segundo ponto informado, ou seja, o algoritmo irá identificar o caminho com o menor custo de deslocamento entre os pontos informados. Como os valores utilizados para a composição da superfície de custo são referentes à probabilidade de existência de linhas, o corredor preferencial será aquele que apresentar a sequência de células da matriz com os maiores valores de probabilidade, portanto para a utilização do algoritmo Least CostlyPathé necessário utilizar como superfície de custo o inverso dos valores de probabilidade.

Como cada pixel das imagens tem uma dimensão de 60 por 60 metros, o algoritmo encontra um corredor de mínimo custo total com 60 metros de largura com maior probabilidade de existência de uma linha de transmissão. Para otimização da rota encontrada a partir do corredor de mínimo custo total, foram inseridas variáveis que aproximem o projeto do objetivo de minimizar custo e impactos. Como os valores utilizados para compor a superfície de custo são referentes ao inverso da probabilidade e estes variam de 0 a 1 , sendo 0 maior probabilidade e 1 menor probabilidade, é possível que as variáveis acrescidas, para otimização da rota, possam receber valores entre 0 e 1 , representando maior ou menor impedância à passagem de linhas de energia. A impedância representa neste trabalho as áreas, cuja utilização da superfície é, em maior ou menor grau, incompatível com a existência de linhas de transporte de energia.

\section{APLICAÇÃO E RESULTADOS DO MODELO}

Após as iterações do modelo proposto foram gerados quatro mapas de probabilidade, um para cada classe de linhas existentes. O mapa final de probabilidade é o resultado da média dos quatro mapas iniciais, pois a média representa o valor mais significativo, considerando todas as classes analisadas. Este mapa final de probabilidade foi utilizado para compor a superfície de custo e identificação dos corredores com custo mínimo total. Conforme a proposta metodológica, os resultados foram submetidos a testes e os valores foram calibrados antes da utilização final.

Nos testes estatísticos realizados o Coeficiente de Cramer e o Joint Information Uncertainty, as variáveis utilizadas não foram consideradas dependentes entre si, visto que nenhuma das comparações par a par ultrapassou o limiar de 0,5 para ambos os testes, conforme proposto por Bonham Carter (1994).

Para o cálculo da probabilidade, conforme as equações de 1 a 6 , é necessário calcular o coeficiente do Peso de Evidência de cada classe das variáveis explicativas do modelo. Este coeficiente representa a influência na maior ou menor probabilidade de existência de linhas de transporte de energia. Para a calibração do modelo foram excluídas as classes das variáveis contínuas que não apresentam uma relação funcional com os coeficientes da regressão utilizada para o cálculo dos Pesos de Evidência. Em outras palavras, foi realizada a exclusão das classes sem interferência na probabilidade de ocorrência de linhas de transmissão de energia. 
A Figura 3 demonstra que antes da exclusão destas classes sem relação funcional com a probabilidade existia a sobreposição de algumas variáveis sobre outras. As cores quentes representam maior probabilidade, enquanto que as frias representam menor probabilidade. No exemplo da Figura 3a, algumas classes de distância aos rios e distância às linhas existentes, sem relação funcional com a probabilidade, foram consideradas e ocasionaram a sobreposição à variável declividade, que neste caso possui uma relação funcional com a probabilidade. $\mathrm{Na}$ Figura 3 b é possível ver uma variação da probabilidade mais ordenada, por exemplo, a baixa probabilidade de existência de linhas de energia no curso do rio e nas suas proximidades, representado pelo elemento linear de cor azul na Figura $3 b$.

Figura 3 - Resultado da calibração do modelo. a) antes da exclusão, b) após exclusão.
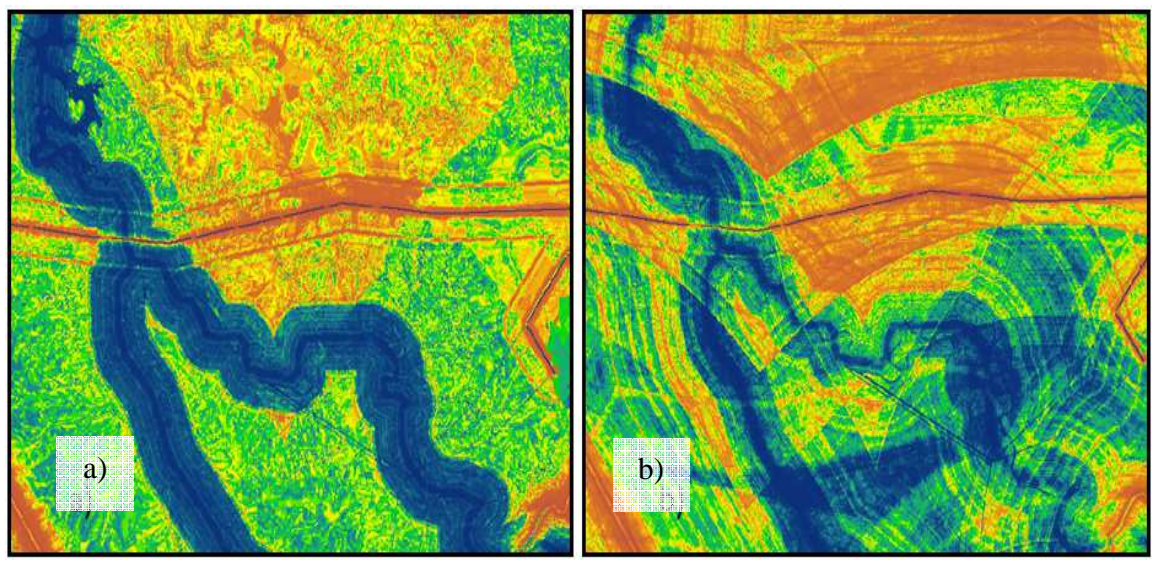

O mapa de probabilidade foi validado traçando a rota de uma linha existente e não considerada no modelo. A linha existente tem a tensão nominal $69 \mathrm{kV}$, foi construída em 2003, com aproximadamente $63,1 \mathrm{~km}$ de extensão e possui uma faixa de domínio de $23 \mathrm{~m}$ de largura. A linha projetada pelo modelo traçou um corredor com $60 \mathrm{~m}$ de largura numa extensão aproximada de 67 km, conforme a Figura 4.

Para a análise de aderência da linha de transmissão projetada pelo modelo à linha já existente foi calculada a distância e o ângulo de cada vértice da linha projetada para à existente. Quase $70 \%$ da linha projetada tem um ângulo que indica paralelismo com a linha existente, sendo que mais de $10 \%$ dos vértices são coincidentes. A distância entre as linhas analisadas indica uma proximidade de até $550 \mathrm{~m}$ para quase $70 \%$ da linha projetada pelo modelo. Para este procedimento foi utilizada a ferramenta do ArcGIS que calcula a menor separação entre feições e o ângulo formado entre o vetor que indica esta separação e a feição de análise, cuja variação está entre $0^{\circ}$ e $180^{\circ}$. 
Figura 4- Resultado da validação do modelo.
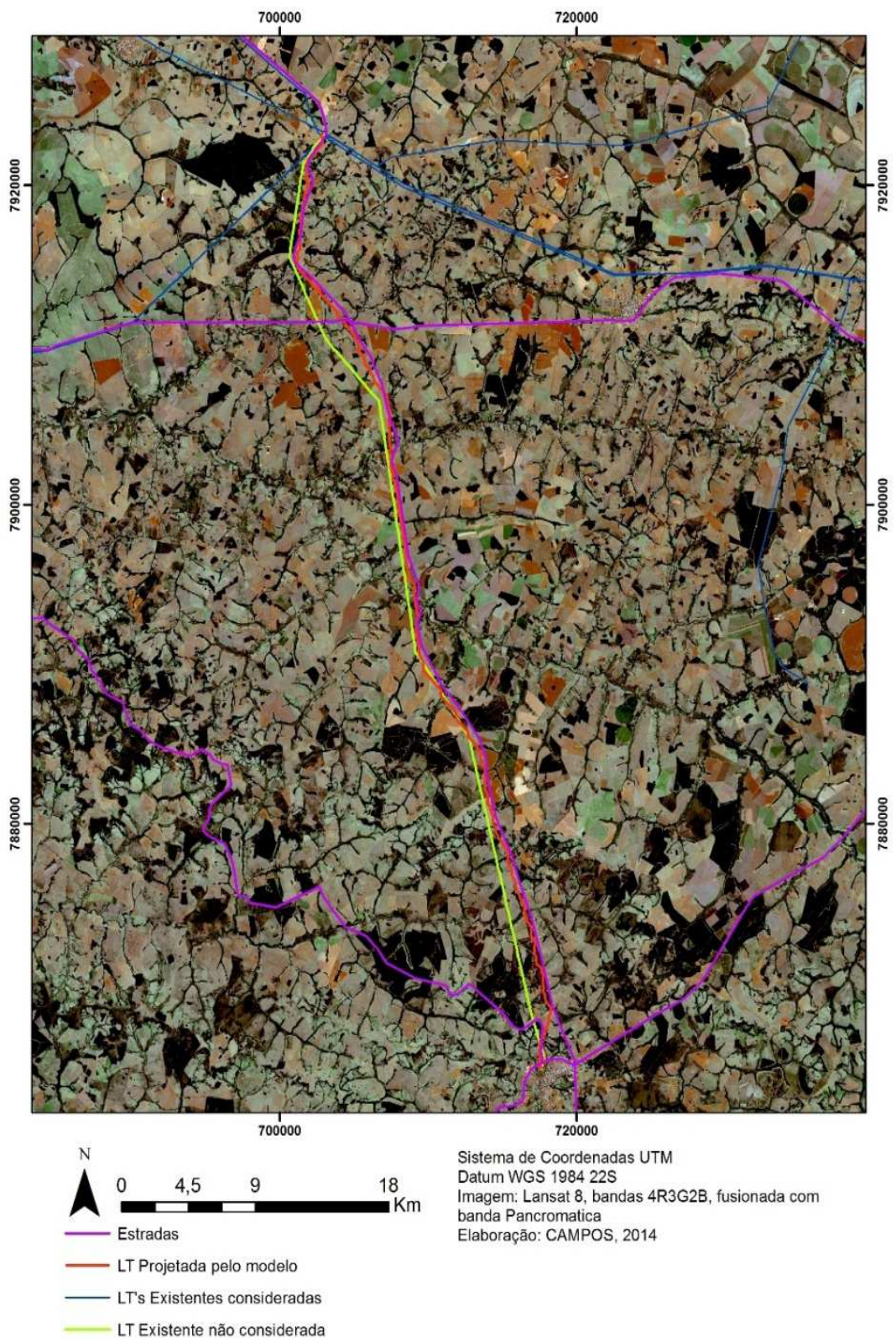

Sistema de Coordenadas UTM

Datum WGS $198422 \mathrm{~S}$

Imagem: Lansat 8, bandas 4R3G2B, fusionada com

banda Pancromatica

Elaboração: CAMPOS, 2014

T Existente não considerada

Bol. Ciênc. Geod. sec. Artigos, Curitiba, v. 20, nº 4, p.765-783, out-dez, 2014. 
Como os traçados são praticamente coincidentes é possível considerar que a validação foi satisfatória para a previsão de um macro corredor preferencial para linhas de transporte de energia. Entretanto, o modelo apresentou algumas variações referentes à distância e ângulo entre a linha projetada pelo modelo e a que foi utilizada para validação. A variação mais significativa está presente próxima ao cruzamento da BR 365, que liga Uberlândia a Montes Claros, com a BR 153, também conhecida como Belém-Brasília, o trevo está localizado no município de Monte Alegre de Minas.

A maior variação neste trecho tem aproximadamente $1,5 \mathrm{~km}$ de distância entre a linha projetada e a linha existente, próximo ao ponto referente ao trevo. Já a análise dos ângulos deste trecho indica que os segmentos estão se distanciando ou seguindo direções diferentes. Este trecho que não indica paralelismo, considerando a análise do ângulo, corresponde a aproximadamente 15\% do traçado da linha projetada.

A Figura 5 apresenta em maior escala este trecho. Analisando em maiores detalhes esta divergência é possível perceber que a linha existente, representada pela cor amarela na Figura 5, desvia de um cruzamento de rodovias, na cor roxa no mapa, e de uma ocupação antrópica da superfície, representada no mapa pela cor laranja, enquanto que a linha modelada acompanha a rodovia sem se sobrepor, mas em sentido oposto à utilizada para a validação. Entretanto, por mais evidente que seja a impedância de um grande cruzamento de rodovias na existência de linhas de energia, em nenhuma das referências consultadas foi identificada uma variável que considerasse este tipo de interferência. Dessa forma, é possível inferir que existe a possibilidade de que algumas variáveis sejam consideradas somente a partir da experiência adquirida ao longo de vários projetos elaborados e executados, uma vez que estas variáveis não estão presentes no referencial normativo do setor.

Para o teste da capacidade de otimização do modelo, uma rota foi traçada considerando apenas o corredor preferencial produto dos Pesos de Evidência, ou seja, considerando a experiência acumulada do modo, até então, preferencial de se projetar e construir linhas de transmissão de energia. A esta mesma rota foram incorporadas as variáveis com grande impedância e não observáveis na paisagem. Conforme a Figura 6, o primeiro traçado proposto pelo modelo (cor vermelha) impacta um assentamento rural (feição em amarelo), enquanto que o segundo traçado (cor roxa) desvia do assentamento rural. 
Figura 5: Detalhe da validação do modelo

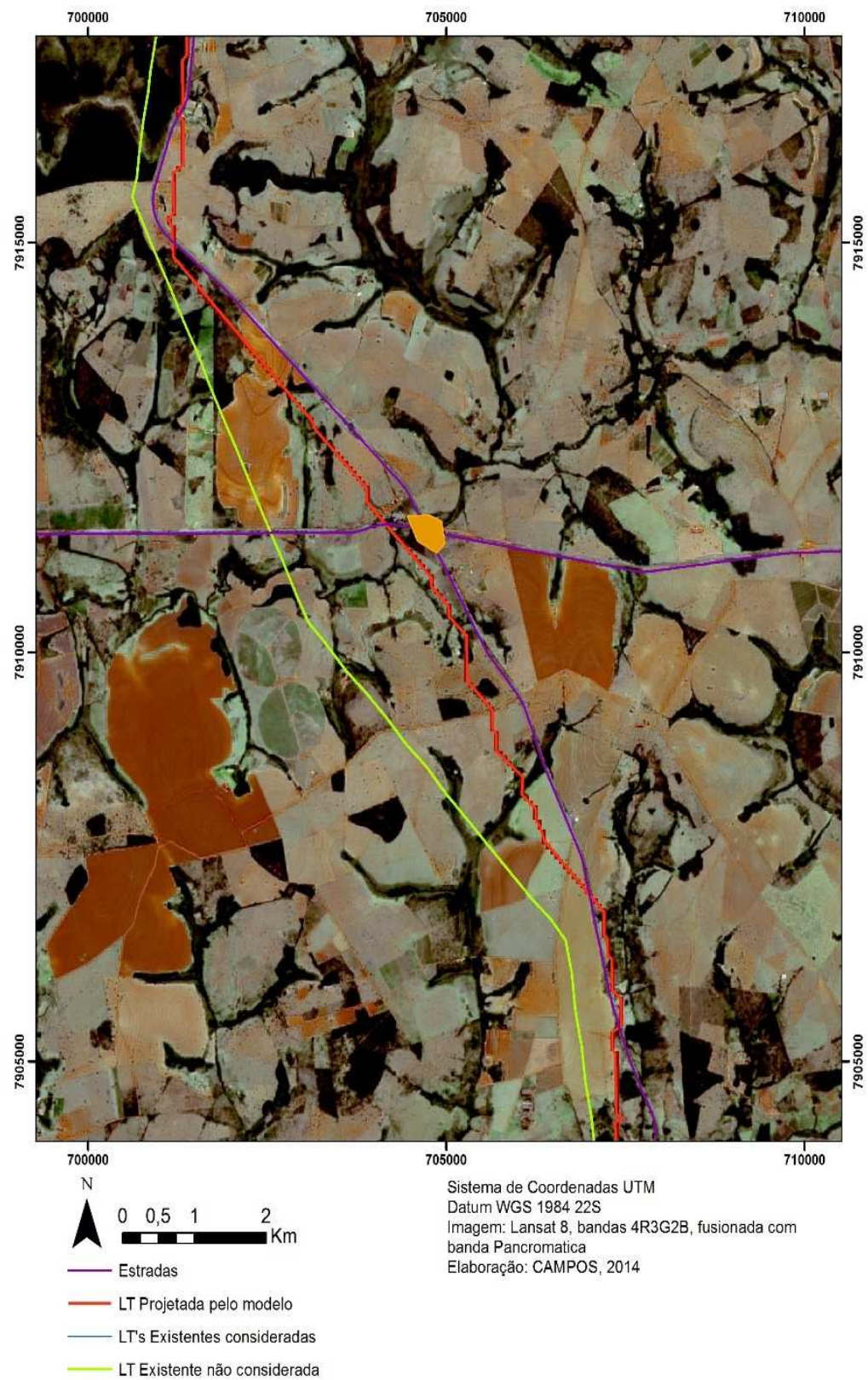

Bol. Ciênc. Geod. sec. Artigos, Curitiba, v. 20, nº4, p.765-783, out-dez, 2014. 
Figura 6: Teste da otimização do modelo.
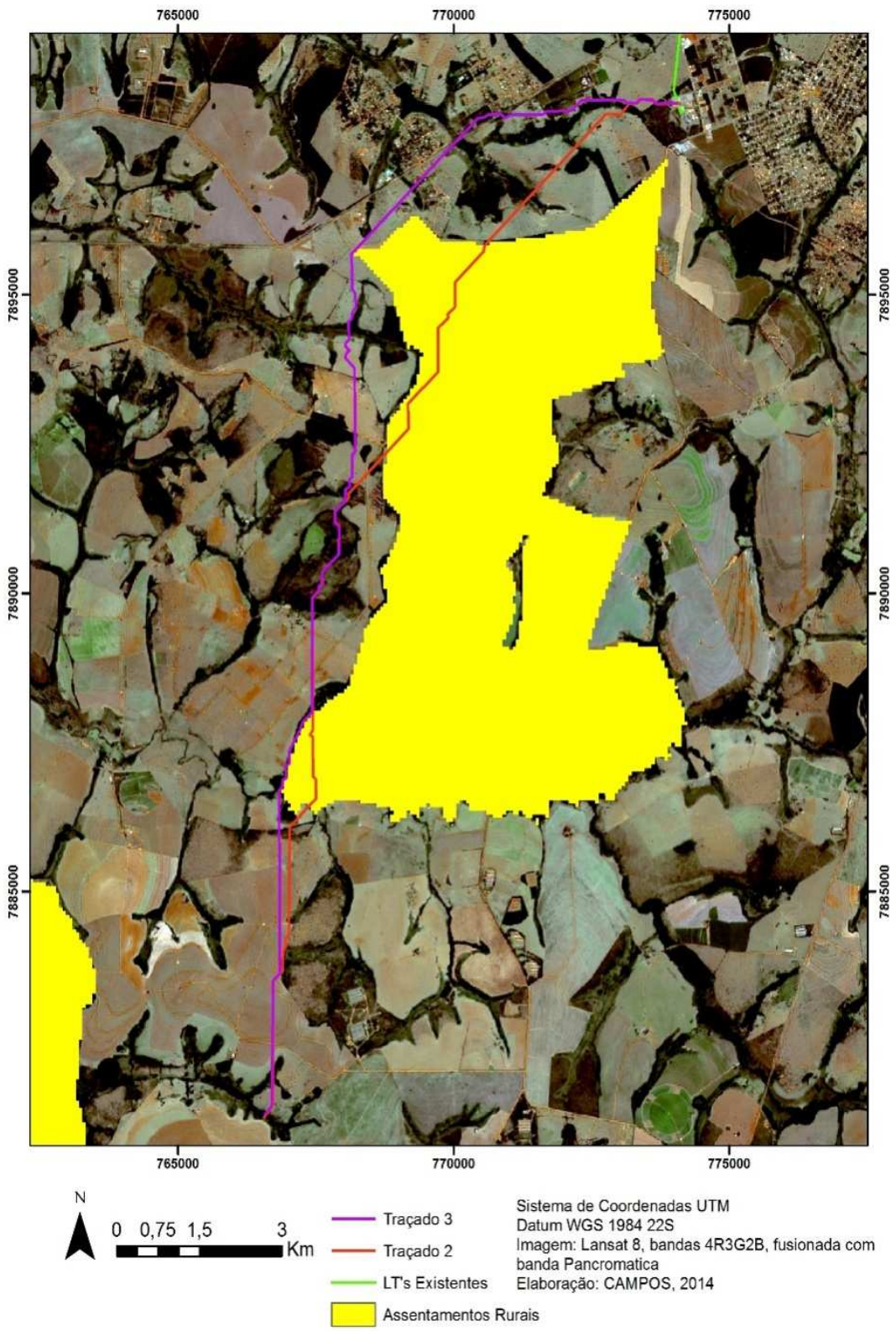

Bol. Ciênc. Geod. sec. Artigos, Curitiba, v. 20, no4, p.765-783, out-dez, 2014. 


\section{CONSIDERAÇÕES FINAIS}

A partir dos resultados apresentados percebe-se que a metodologia proposta pôde identificar os padrões espaciais das feições lineares que representam as linhas de transmissão de energia elétrica. Estes padrões espaciais foram utilizados como variáveis explicativas na função de inferência da probabilidade de existência destas feições na paisagem. Utilizando esta probabilidade, o modelo apresentado foi capaz de prever rotas muito próximas para as linhas de energia que já existiam, possibilitando dessa forma utilizar o modelo para prever rotas de projetos futuros.

$\mathrm{O}$ teste de validação do modelo foi satisfatório ao apresentar uma aderência de mais de $70 \%$ da rota modelada à rota já existente. As diferenças encontradas no teste de validação evidenciam que o projeto executivo tem um refinamento na locação do traçado que a escala abordada nesse estudo não abrange. Entretanto, esta divergência mostra outro ponto importante que é a possibilidade de identificação de outras variáveis explicativas do modelo e de conhecimentos não considerados nas referências de fundamentação existentes na literatura do setor.

Além disso, a característica multitemporal do modelo implica na inserção da evolução destes padrões espaciais na capacidade de inferência do modelo. No estudo de caso, foi possível perceber a preferência para locação de linhas de transporte de energia elétrica quando associada à experiência de mais de vinte e cinco anos de projetos de uma mesma empresa.

Já a proposta de otimização das rotas traçadas pelo modelo mostra a possibilidade de verificação do impacto de um desvio do traçado ainda na fase de planejamento. Em outras palavras, a utilização da abordagem multicritério possibilita apontar rotas alternativas que são passiveis de serem comparadas e que auxiliam a decisão, considerando de forma conjunta, questões a princípio conflitantes.

Propõem-se em continuidade à pesquisa a aplicação do modelo em outras regiões, com características diferentes da estudada. Dessa forma será possível estabelecer se é mantida a consistência para as variáveis utilizadas ou se existe a necessidade de calibração do modelo para cada tipo de região. Pode-se ainda utilizar a metodologia proposta para avaliação dos planos de expansão do setor elétrico, uma vez que a identificação dos corredores preferenciais à ocorrência de linhas de transmissão de energia pode indicar o impacto provável da forma preferencial de se implantar este tipo de empreendimento, e ainda apontar alternativas que minimizem os impactos negativos.

\section{REFERÊNCIAS BIBLIOGRÁFICAS}

ABNT. Projeto de Linhas Aéreas de Transmissão de Energia Elétrica (NBR 5.422). Rio de Janeiro: ABNT, 1985.

AGTERBERG, F.P.; BONHAM-CARTER, G.F. Deriving weights of evidence from geosciences contour maps for the prediction of discrete events. XXII Int. Symposium AP-COM, p. 381-395,1990. 
ALMEIDA, C. M.; MONTEIRO, A. M. V.; CÂMARA G. Stochastic cellular automata modeling of urban land use dynamics: empirical development and estimation. Computers, Environment and Urban Systems, New York, v. 27, n. 5, p. 481-509, 2003.

ANEEL - Procedimentos de Distribuição de Energia Elétrica no Sistema Elétrico Nacional -PRODIST. Agência Nacional de Energia Elétrica, 2013.

BELÉM, F. L.; FONSECA, A. R.; PIMENTA L.C.A.; REZENDE D.T.; TAVARES T.H.B.C.; MESQUITA R.C. Ferramenta para seleção de corredor de linha aérea de transmissão utilizando geoprocessamento. XIV Simpósio Brasileiro de Sensoriamento Remoto, Natal. Anais dos XIV SBSR - Simpósio Brasileiro de Sensoriamento Remoto. São Jose dos Campos: Ed Inpe,v. 1. p. 3559-3566, 2009.

BONHAM-CARTER, G. F. Geographic information systems for geoscientists: modeling with GIS. New York: Pergamon,1994.

CHORLEY, R.J.; HAGGET, P. Modelos Integrados em Geografia. Rio de Janeiro, Editora Livros Técnicos e Científicos S.A., p.279, 1974.

CHRISTOFOLETTI, A. Modelagem de Sistemas Ambientais. São Paulo: Edgar Blücher, p. 236,1998.

EPRI. Overhead Electric Transmission Line Siting Methodology. EPRI, Palo Alto, CA, and Georgia Transmission Corporation, Tucker, GA, 2006.

LABEGALINI, P. R.; LABEGALINI, J. A.; FUCHS, R. D.;ALMEIDA, M. T. Projetos Mecânicos das Linhas Aéreas de Transmissão, 2a. Edgard Blücher, 1992.

MENDES, C. A. B.; CANALES, F. A. V. Técnicas de regressão logística aplicada a análise ambiental. Geografia (Londrina), v. 20, p. 5-30, 2011.

MUNIER, N.; A Strategy for Using Multicriteria Analysis in Decision-Making: A Guide for Simple and Complex Environmental Projects. Springer, New York, 2011.

NÓBREGA, R. A. A. Understanding Spatial-Criteria decision making: An analytical demonstration of AHP-based MCDM and how it is used in GIS. National Consortium for Remote Sensing in Transportation (NCRST). Annual Workshop. Memphis: NCRST / Geosystems Research Institute, 2009.

NUNES, F.S.M. Valoração florestal através de modelagem da rentabilidade da extração de castanha-do-brasil em Madre de Dios, Peru. Brasil. Tese, Mestrado em Análise e Modelagem de Sistemas Ambientais. Universidade Federal de Minas Gerais,2010.

POLANYI, M..The tacit dimension. London. Routledge \& Kegan Paul, 1966.

RIBEIRO, R. Tacit knowledge management. Phenomenology and the Cognitive Sciences (Impresso), v. 11, p. 1-30, 2012.

MACEDO, R. C.; ALMEIDA, C. M.; SANTOS, J. R.; RUDORFF, B. F. T. Modelagem dinâmica espacial das alterações de cobertura e uso da terra relacionadas à expansão canavieira. Boletim de Ciências Geodésicas (Online), v. 19, p. 313-337, 2013. 
SADASIVUNI, R.; C. G. O’HARA.; R. A. A. NOBREGA.; J. DUMAS.A transportation corridor case study for Multi- Criteria decision analysis. In Proceedings: American Society of Photogrammetry and Remote Sensing Annual Conference. Baltimore, MD, 2009.

SAATY, T.L. The Analytic Hierarchy Process. McGraw-Hill, New York, NY. 1980 SCHMIDT, A. J. Implementing a GIS Methodology for Siting High Voltage Electric Transmission Lines.Resource Analysis. Saint Mary's University of Minnesota University Central Services Pressv. 11, p. 17, 2009.

SOARES-FILHO, B. S. Modelagem da dinâmica de paisagem de uma região de fronteira de colonização amazônica. Tese, Doutorado em Engenharia Civil, Escola Politécnica da Universidade de São Paulo (EPUSP). São Paulo, 1998.

SOARES-FILHO, B.S; RODRIGUES, H.O; FALIERI, A; COSTA, W. L., Dinâmica EGO, 2008. Tutorial http://www.csr.ufmg.br/dinamica

((Recebido em fevereiro de 2014. Aceito em julho de 2014). 